\title{
Using Ionic Liquids for the Separation of Carbohydrates
}

\author{
Mohamed K. Hadj-Kali and Inas M. AlNashef
}

\begin{abstract}
Ionic liquids (ILs) were used to separate fructose and glucose from their mixtures at different temperatures. It was found that the solubility of glucose and fructose in ILs depends on the type of the anion, cation, and substituents on the cation; with the anion playing the most important role. Depending on the type of the anion, some ILs dissolve more of one sugar than the other. A separation process resulting in either precipitation or enriched solutions of either of the two sugars was proposed. More specifically, the process uses ILs, as selective solvents, and applies dissolution and filtration to separate the precipitated sugar. Separation of the sugars from the IL is then conducted by extraction with water in a centrifuge. The IL is then recycled. The recovery of sugars in all cases was higher than $90 \%$ and the purity of the separated sugar was $99 \%$. The solubility of glucose in different ionic liquids is estimated using the NRTL activity coefficient model.
\end{abstract}

Index Terms-Ionic liquids, separation process, sugars.

\section{INTRODUCTION}

Biomass in general and carbohydrates in particular are very valuable, abundant and renewable feedstocks for the production of chemicals and biofuels [1]-[3]. The traditional sweetener in the food industry is sucrose, however, high fructose corn syrups (HFCS) replaced sucrose in many applications due to their superior sweetening properties (about 1.3-1.8 times that of sucrose) and higher solubility especially at lower temperatures. In the production of high fructose syrups, an important step is the separation of fructose from its mixtures with glucose [4]. In many cases pure sugars (glucose or fructose) are required, for instance, the food industry utilizes large quantities of high fructose corn syrup while pure glucose is used for medical purposes and in the manufacture of pharmaceuticals.

Glucose and fructose, being isomers, are difficult and costly to separate. Chromatography is the commercial method used for sugar separations. It is currently applied to enrich fructose content in HFCS [5]. As a batch process, the method suffers from low productivity and low yields of the desired product and normally requires expensive installations. Attempts to simulate continuous operation were hampered by the complexity of the process and the associated equipment and the high operation costs [2]-[6]. Simulated moving bed processes have been frequently applied in the carbohydrate industry, where they are used for the production of fructose-enriched HFCS and for the recovery of sucrose from molasses [7]. Limited successes obtained with these

Manuscript received November 20, 2014; revised March 15, 2015. This work was supported by the Deanship of Scientific Research at King Saud University through the Research Group Project no. RGP-VPP-108.

The authors are with the Chemical Engineering Department, King Saud University, P.O. Box 800, 11421, Riyadh, KSA (e-mail: mhadjkali@ksu.edu.sa). processes reveal the need to develop more efficient processes.

Ionic liquids (ILs), which have been widely promoted as "green solvents", are attracting much attention for applications in many fields of chemistry and industry due to their chemical stability, thermal stability, low vapor pressure and high ionic conductivity properties.

Lau et al. were the first to explore the potential use of ILs as media for carbohydrate transformation [8]. It was then reported that the dicyanamide is an attractive anion to dissolve carbohydrates, due to its hydrogen bond acceptor properties [9]-[12]. N, N-methylmethoxyalkylimidazolium was reported to have a sugar-philic cation, rather than anion [10]-[13]. Liu et al. found that ILs containing the dicynamide anion dissolved glucose more than an order of magnitude higher than their tetrafluoroborate counterparts. The solubility of glucose in 1-butyl-3-methylimidazolium dicynamide at $25^{\circ} \mathrm{C}$ was measured to be $145 \mathrm{~g} / \mathrm{L} \mathrm{[14].}$ Rosatella et al. performed an extended study on the solubility of the carbohydrates glucose, fructose, sucrose and lactose in twenty eight different ILs. They reported that it was possible to achieve solubilities, at $35^{\circ} \mathrm{C}$, of each carbohydrate up to 43.9, 49.0, 17.1 and 16.6 ( $\mathrm{g}$ of carbohydrate per $100 \mathrm{~g}$ of IL), respectively [15]. Zhao et al. found that ether-functionalized ILs can dissolve considerable amounts of D-glucose and cellulose [16], [17].

In this paper the utilization of ionic liquids for separating glucose and fructose from their mixture is addressed. The dissolution capacity of ionic liquids for individual sugars and mixed sugars is determined at room temperature. The paper addresses also the effect of temperature on the dissolution of mixed sugars in selected ionic liquids. A procedure for extracting the dissolved sugars from the ionic liquids will be outlined. The preliminary calculations of glucose solubilities in different ionic liquids using the NRTL model are also reported.

\section{MAterials AND MethodS}

\section{A. Chemicals}

TABLE I: LIST OF IONIC LIQUIDS USED IN THIS WORK

\begin{tabular}{|l|l|l|}
\hline Ionic Liquid & Nomenclature & $A b v$. \\
\hline $\begin{array}{l}\text { 1-Ethyl-3-methylimidazolium } \\
\text { dicyanamide }\end{array}$ & {$\left[\right.$ EMIM] $\left[\mathrm{N}(\mathrm{CN})_{2}\right]$} & IL1 \\
\hline $\begin{array}{l}\text { 1-Ethyl-3-methylimidazolium } \\
\text { ethylsulfate }\end{array}$ & {$[\mathrm{EMIM}]\left[\mathrm{C}_{2} \mathrm{H}_{5} \mathrm{OSO}_{3}\right]$} & IL2 \\
\hline $\begin{array}{l}\text { 1-Ethyl-3-methylimidazolium } \\
\text { n-hexylsulfate }\end{array}$ & {$\left[\right.$ EMIM] $\left[\mathrm{C}_{6} \mathrm{H}_{13} \mathrm{OSO}_{3}\right]$} & IL3 \\
\hline $\begin{array}{l}\text { 1-Ethyl-3-methylpyridinium } \\
\text { ethylsulfate }\end{array}$ & {$[\mathrm{EMPY}]\left[\mathrm{C}_{2} \mathrm{H}_{5} \mathrm{OSO}_{3}\right]$} & IL4 \\
\hline $\begin{array}{l}\text { Dimethylimidazolium } \\
\text { dimethylphosphate }\end{array}$ & $\left.[\mathrm{MMIM}]\left[\mathrm{CH}_{3} \mathrm{O}\right)_{2} \mathrm{PO}_{2}\right]$ & IL5 \\
\hline
\end{tabular}


D-glucose and D-fructose (purity $>99 \%$ ) were purchased from Winlab Co. (UK). All ILs (purity >98\%) were procured from Solvent Innovation GmbH (Köln, Germany). More than fifteen ILs were tested for the separation of glucose and fructose from their mixture. However, in this paper we summarized the results obtained for only five ILs that are listed in Table I.

\section{B. Experimental Setup}

All chemicals were of $>99 \%$ purity and were used without further purification. Dissolution experiments were performed in a bench-top shaking incubator with $\pm 0.1{ }^{\circ} \mathrm{C}$ accuracy. Typically, $6 \mathrm{~g}$ of IL were added in a $20 \mathrm{~mL}$ capped vial. Small amounts of glucose or/and fructose were added batchwise to the vial and allowed to dissolve under continuous shaking at constant temperature. When the IL solution turned clear, more glucose or/and fructose was added. When the IL solution did not become clear after several hours of sugar addition, samples from the solution were taken, filtered, dissolved in HPLC grade acetonitrile and then analyzed using high performance liquid chromatography, HPLC. The IL is considered saturated with a sugar when two samples, taken within hours from each other, give approximately the same concentration of the sugar. The average total time for a dissolution experiment is 2 weeks. The undissolved solid was removed by centrifuging at $13600 \mathrm{rpm}$ for several minutes (Eppendorf modal 5415D, Germany). The resulting IL solution was immediately dissolved in acetonitrile for HPLC analysis. It should be noted that when both glucose and fructose are dissolved in the same IL, the IL may become saturated with one of the sugars well before the other. The data reported here is the average value of at least triplicate runs.

\section{RESULTS AND DISCUSSION}

The strategy adopted here was to measure the solubility of glucose and fructose separately in ILs, i.e. either glucose or fructose is added to the IL, in different ILs. The results are shown in Table II.

TABLE II: Solubility of Glucose AND Fructose, MEAsured

\begin{tabular}{|c|c|c|c|}
\hline \multicolumn{4}{|c|}{ SEPARATELY, AT $25^{\circ} \mathrm{C}$} \\
\hline \multirow{2}{*}{ ILs } & \multicolumn{2}{|c|}{ Solubility (g/100g IL) } & \multirow{2}{*}{$\mathrm{F} / \mathrm{G}$} \\
\hline & G & $\mathrm{F}$ & \\
\hline IL1 & 33.9 & 60.6 & 1.79 \\
\hline IL2 & 30.9 & 56.4 & 1.83 \\
\hline IL3 & 8.1 & 25.2 & 3.11 \\
\hline IL4 & 11.6 & 12.6 & 1.09 \\
\hline IL5 & 50.2 & 25.6 & 0.51 \\
\hline
\end{tabular}

It is clear from Table II that for the sulfate anion based ILs (i.e., IL2, IL3, IL4) the solubility of fructose is always higher than that for glucose under the same conditions. The capacity of the IL to dissolve fructose decreased in the order of IL2>IL3>IL4. On the other hand, the solubility of fructose is much smaller than that for glucose for the phosphate anion based ILs (IL5). It is interesting to note that the solubility of glucose and fructose in IL4 was quite similar $(\mathrm{F} / \mathrm{G}=1.09)$. The dissolution capacity of the dicyanamide-based ionic liquid (IL1) for fructose is higher than that of the sulfate-based ILs. Similar results were reported in the literature but for different types of ILs. For example, Lee et al. [18] found that the solubility of fructose in ethylmethylimidazolium ethylmethylimidazolium tetrafluoroborate, trifluorosulfonate, butylmethylimidazolium tetrafluoroborate and butylmethylimidazolium trifluorosulfonate, at $25^{\circ} \mathrm{C}$ is $7.7,32.8,3.3$, and $27 \mathrm{~g} / \mathrm{L}$, respectively, while the solubility of glucose under the same conditions was $1.1,6.1,0.9$, and $4.8 \mathrm{~g} / \mathrm{L}$, respectively. It is clear that the effect of the anion on the extent of solubility is stronger than that of the cation. This anion effect was confirmed by Rosatella et al. [15] who reported that the solubility of glucose in methoxyethoxyethylmethylimidazolium (-chloride and -dicynamide) at $35^{\circ} \mathrm{C}$ was 29.28 and $19.01 \mathrm{~g} / 100 \mathrm{~g}$, respectively, while the solubility of fructose under the same conditions was 14.10 and 48.99 $\mathrm{g} / 100 \mathrm{~g}$, respectively.

Since the main objective of this work was to investigate the possibility of using ILs for the separation of fructose and glucose from their solid mixtures, the next step was to measure the solubility of fructose and glucose when they are both present in the IL. In order to study the effect of anion and cation of the ILs, the solubility of fructose and glucose was measured at $25^{\circ} \mathrm{C}$. The results are shown in Table III. Compared to the single sugar experiments, in most cases the solubility of fructose was affected by the presence of glucose and vice versa. As shown in Table III, the solubility of fructose is still higher than that for glucose under the same conditions for the sulfate anion based ILs (IL2, IL3 and IL4) and the reverse is true for phosphate-based IL (IL5). It can be noted that the anion, cation, substituents on the cation, and substituents on the anion affected the solubility of sugars in the tested ILs. The effect of changing the anion or the cation alone can be appreciated when the solubility of glucose $(\mathrm{G})$ in IL2 and IL4 (for the cation) are compared, for example. It is clear from Table III that the solubility of glucose dropped upon changing the cation from 20.1 for IL2 (1-ethyl-3-methylimidazolium) to $8.8 \mathrm{~g} / 100 \mathrm{~g}$ for IL4 (1-ethyl-3-methylpyridinium).

It is also clear that the solubility of fructose in IL2-IL4 (mostly sulfate-based) is more than that of glucose $(\mathrm{F} / \mathrm{G}>1)$. This group is called type A ILs, while the more glucose-dissolving group (IL5) is called type B ILs $(\mathrm{F} / \mathrm{G}<1)$. It was found that the imide based ILs have very limited dissolution capacity (regardless of $\mathrm{F} / \mathrm{G}$ values); therefore they were not further investigated.

TABLE III: SolubiLITy OF GLUCOSE AND FRUCTOSE, MEASURED Mutually, AT $25^{\circ} \mathrm{C}$

\begin{tabular}{|c|c|c|c|}
\hline \multirow{2}{*}{ ILs } & \multicolumn{2}{|c|}{ Solubility (g/100g IL) } & \multirow{2}{*}{$\mathrm{F} / \mathrm{G}$} \\
\hline & $\mathrm{G}$ & $\mathrm{F}$ & \\
\hline IL1 & 20.20 & 57.40 & 2.84 \\
\hline IL2 & 20.10 & 55.80 & 2.78 \\
\hline IL3 & 11.20 & 23.90 & 2.13 \\
\hline IL4 & 8.80 & 10.10 & 1.15 \\
\hline IL5 & 75.00 & 44.80 & 0.60 \\
\hline
\end{tabular}

In order to show the validity of the above classification, IL2 from type A and IL5 from type B were used. A 1:1 mixture of fructose and glucose was added to the ionic liquid at room temperature. Based on results of previous 
experiments listed in Table III, the total amount of the mixture was adjusted so that one sugar dissolves completely in the corresponding IL (i.e., fructose for IL2 and glucose for IL5) while some of the other sugar preciptates. The sugar-saturated IL solution was separated from the precipitate for both cases by normal filtration. HPLC chromatograms for the precipitate from the two cases are shown in Fig. 1 (Fig. 1.a for IL2 and Fig. 1.b for IL5). It is clear from Fig. 1.a that no fructose is present in the precipitate (i.e., all fructose had dissolved in IL2) and only glucose is present while Fig. 1.b reveals the opposite for IL5. This finding confirms the above classification (i.e., glucose from type A ILs, or fructose from type B ILs, with a purity of more than $99 \%$ can be separated from the saturated IL using centrifuging and/or filtration). Taking into consideration the close similarity in the chemical structure of glucose and fructose (being isomers) the aforementioned results prove that ILs can be used for the separation of compounds that are very difficult to separate using conventional methods.
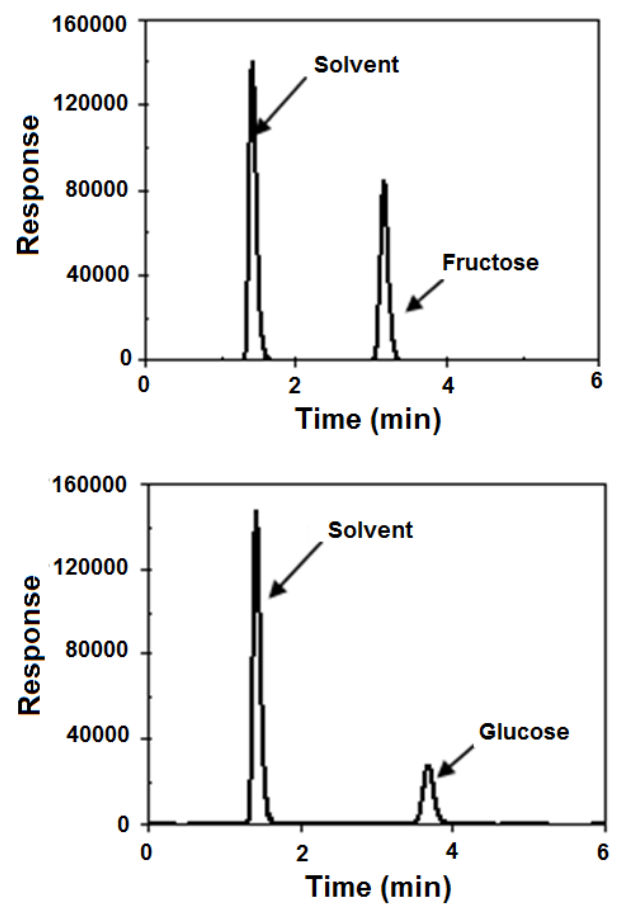

Fig. 1. HPLC chromatograms of the precipitates of IL2 (in the top) and IL5.

In order to increase the dissolution rate and to study the effect of temperature on the solubility of fructose and glucose, the solubility of glucose and fructose in selected ILs at different temperatures was measured. It is worth-noting that the solubility experiments were conducted by the addition of an excess amount of sugars to the ILs at the studied temperature, and not by dissolution at higher temperature followed by cooling down to the desired temperature, thus the possibility of dissolution of sugars under supersaturated conditions was avoided [15]. Before taking a sample, the IL saturated with sugars was centrifuged for several minutes at 13,600 RPM in order to be sure that no crystal of sugars are dispersed in the IL. Three ionic liquids; two of Type A (IL2 and IL4) and one of Type B (IL5) were tested.

As seen from Table IV, the solubility of both fructose and glucose in all ILs increased with the increase of temperature. For IL2 and IL4, the ratio of fructose to glucose decreased with increasing temperature, thus indicating the higher enhancement of glucose solubility compared to that of fructose. The opposite is true for IL5. While the solubility of fructose continued to increase with temperature for IL4, the glucose solubility passed through a maximum solubility at 50 ${ }^{\circ} \mathrm{C}$.

Table IV reveals also that the dissolution capacity of ILs is much higher for Imidazolium-based ILs (IL2, IL5) compared to pyridinium-based (IL4). In addition, it is interesting to note that, for IL4 temperatures below $55{ }^{\circ} \mathrm{C}$ the solubility of fructose is higher than that for glucose. The opposite is true for temperatures above $55{ }^{\circ} \mathrm{C}$. Appreciating the dramatic decrease of viscosity of ILs with the increase of temperature, it is obvious that it is better to perform the separation process at $50-60{ }^{\circ} \mathrm{C}$. This will reduce the power needed for mixing, reduce the time needed to reach equilibrium, and increases the amount of sugars that can be separated per unit mass of IL. However, the cost of energy needed for heating should be taken into consideration.

TABLE IV: SOLUBILITY OF GLUCOSE AND FRUCTOSE, MEASURED Mutually, AT $25^{\circ} \mathrm{C}$.

\begin{tabular}{|c|c|c|c|c|}
\hline \multicolumn{5}{|c|}{ MUTUALLY, AT $25^{\circ} \mathrm{C}$. } \\
\hline & \multirow{2}{*}{$\mathrm{T}\left({ }^{\circ} \mathrm{C}\right)$} & \multicolumn{2}{|c|}{ Solubility (g/100g IL) } & \multirow{2}{*}{$\mathrm{F} / \mathrm{G}$} \\
\hline & & $\mathrm{G}$ & $\mathrm{F}$ & \\
\hline \multirow{4}{*}{ IL2 } & 25 & 20.1 & 55.8 & 2.78 \\
\hline & 40 & 31.7 & 76.6 & 2.42 \\
\hline & 50 & 33.6 & 86.3 & 2.57 \\
\hline & 60 & 48.6 & 89.5 & 1.84 \\
\hline \multirow{5}{*}{ IL4 } & 25 & 8.8 & 10.1 & 1.15 \\
\hline & 40 & 9.7 & 12.1 & 1.25 \\
\hline & 50 & 11.8 & 12.6 & 1.07 \\
\hline & 60 & 14.6 & 5.9 & 0.40 \\
\hline & 70 & 14.7 & 6.0 & 0.40 \\
\hline \multirow{4}{*}{ IL5 } & 25 & 75.0 & 44.8 & 0.60 \\
\hline & 40 & 81.6 & 58.0 & 0.71 \\
\hline & 50 & 83.4 & 60.0 & 0.72 \\
\hline & 60 & 90.0 & 62.0 & 0.69 \\
\hline
\end{tabular}

\section{SOLUBILITY MODELING}

\section{A. Thermodynamics Background}

The basic equation for predicting the saturation mole fraction of a solid in a liquid is [19]-[20]:

$$
\ln \left(x_{1} \gamma_{1}\right)=-\frac{\Delta \underline{H}^{f u s}\left(T_{m}\right)}{R T}\left[1-\frac{T}{T_{m}}\right]-\frac{1}{R T} \int_{T_{m}}^{T} \Delta C_{P} d T+\frac{1}{R} \int_{T_{m}}^{T} \frac{\Delta C_{P}}{T} d T
$$

The subscript 1 denotes the solid solute, $x_{1}$ and $\gamma_{1}$ respectively its molar composition (solubility at equilibrium) and activity coefficient in the mixture, $T_{m}$, the melting point temperature, $T$, the temperature of the system at equilibrium, $\Delta H^{f u s}$ and $\Delta C_{P}$ the enthalpy and heat capacity changes from the solid to the liquid state of the solute.

Two approximations can be made without introducing appreciable error:

1) First, we assume that $\Delta C_{P}$ is independent of temperature. So that the last equation becomes:

$$
\ln \left(x_{1} \gamma_{1}\right)=-\left\{\frac{\Delta \underline{H}^{f u s}\left(T_{m}\right)}{R T}\left[1-\frac{T}{T_{m}}\right]+\frac{\Delta C_{P}}{R T}\left[1-\frac{T_{m}}{T}+\ln \left(\frac{T_{m}}{T}\right)\right]\right\}
$$


2) Then, since the melting point temperature $\left(T_{m}\right)$ at any pressure and the triple point temperature $\left(T_{r}\right)$ are only slightly different for most solids, we can rewrite the last equation, without much error, as:

$$
\ln x_{1}=-\ln \gamma_{1}-\left\{\frac{\Delta H^{f u s}\left(T_{r}\right)}{R T}\left[1-\frac{T}{T_{r}}\right]+\frac{\Delta C_{P}}{R T}\left[1-\frac{T_{r}}{T}+\ln \left(\frac{T_{r}}{T}\right)\right]\right\}
$$

If the liquid mixture is ideal, $\gamma_{l}=1$ and the solubility can be computed from only the thermodynamic data $\left(\Delta H^{f u s}\right.$ and $\Delta C_{P}$ ) for the solid species near the melting point.

For non-ideal solutions, $\gamma_{1}$ must be estimated from either experimental data or liquid solution models, like UNIFAC model.

This equation may be used assuming the simple eutectic mixtures with full miscibility in the liquid and immiscibility in the solid phases. But, because of the lack of appropriate data representing the difference $\Delta C_{P}$ between the heat capacities of the solute in the solid and the liquid states for the systems containing ionic liquids (especially for those systems where the ionic liquid represents the solid phase), the simplified version of the solubility without the $\Delta C_{P}$ term was applied:

$$
\ln x_{1}=-\ln \gamma_{1}-\left\{\frac{\Delta \underline{H}^{f u s}\left(T_{r}\right)}{R T}\left[1-\frac{T}{T_{r}}\right]\right\}
$$

The expected error as an effect of neglecting $\Delta C_{P}$ usually depends on the substance. For normal molecular compounds, the error does not exceed $2 \%$.

The physical properties of the pure glucose and fructose are shown in Table V. The melting temperature and enthalpy of fusion are taken from the Dortmund Data Bank (http://www.ddbst.com/) while the heat capacity differences are those estimated by Ferreira et al. [21].

TABLE V: GLUCOSE AND FRUCTOSE PHYSICAL PROPERTIES
\begin{tabular}{|l|c|c|}
\hline & Glucose & Fructose \\
\hline$\Delta H^{f u s}(\mathrm{~J} / \mathrm{mol})$ & $31410[21]$ & $36005[21]$ \\
\hline$T_{m}(\mathrm{~K})$ & $419.15[21]$ & $377.15[21]$ \\
\hline$\Delta C_{p}(\mathrm{~J} /(\mathrm{mol} \mathrm{K}))$ & $120.00[22]$ & $120.00[22]$ \\
\hline
\end{tabular}

\section{B. NRTL Model}

The Non-Random Two Liquid (NRTL) model [22] is an activity coefficient model frequently applied in the field of chemical engineering to calculate phase equilibrium. In this model, within a liquid solution, local compositions are presumed to account for the short range order and non-random molecular orientations that result from differences in molecular sizes and intermolecular forces.

For a multi-component system, NRTL equation expressed in terms of activity coefficients is:

with

$$
\ln \gamma_{i}=\frac{\sum_{j} \tau_{j i} G_{j i} x_{j}}{\sum_{j} G_{j i} x_{j}}+\sum_{j} \frac{G_{i j} x_{j}}{\sum_{k} G_{k j} x_{k}}\left(\tau_{i j}-\frac{\sum_{k} \tau_{k j} G_{k j} x_{k}}{\sum_{k} G_{k j} x_{k}}\right)
$$

$$
\ln G_{i j}=-\alpha_{i j} \tau_{i j} \quad \alpha_{i j}=\alpha_{j i} \quad \tau_{i i}=0
$$

This equation has three parameters, $\tau_{i j}, \tau_{j i}$ and $\alpha_{i j}$, for each pair of components in the multi-component mixture. So, the
NRTL equation expressed for a binary system gives:

$$
\ln \gamma_{1}=x_{2}^{2}\left(\tau_{21}\left(\frac{G_{21}}{x_{1}+x_{2} G_{21}}\right)^{2}+\frac{\tau_{12} G_{12}}{\left(x_{2}+x_{1} G_{12}\right)^{2}}\right)
$$

The model development is achieved within Simulis ${ }^{\circledR}$ Thermodynamics environment, a thermo physical properties calculation server provided by ProSim company (http://www.prosim.net/) as an MS-Excel add-in.

The so-called non-randomness parameter $\alpha_{12}$ is taken equal to 0.20 as usual. Then, assuming that the solubility of glucose is not affected by fructose, the binary interaction parameters $\tau_{i j}$ and $\tau_{j i}$ are estimated from the $N$ experimental data points of Table IV for each "pseudo-binary" mixture (glucose-IL). An iterative process is used at each temperature and minimizing the quadratic relative criterion between calculated and experimental solubility:

$$
\text { Criterion }=\frac{1}{N} \sum_{N}\left(\frac{x_{1}{ }^{e x p}-x_{1}{ }^{c a l}}{x_{1}{ }^{e x p}}\right)^{2}
$$

Linear temperature dependence is obtained for the binary interaction parameters. Comparison of the model with experimental data is presented in Fig. 2 for IL2 and IL4.
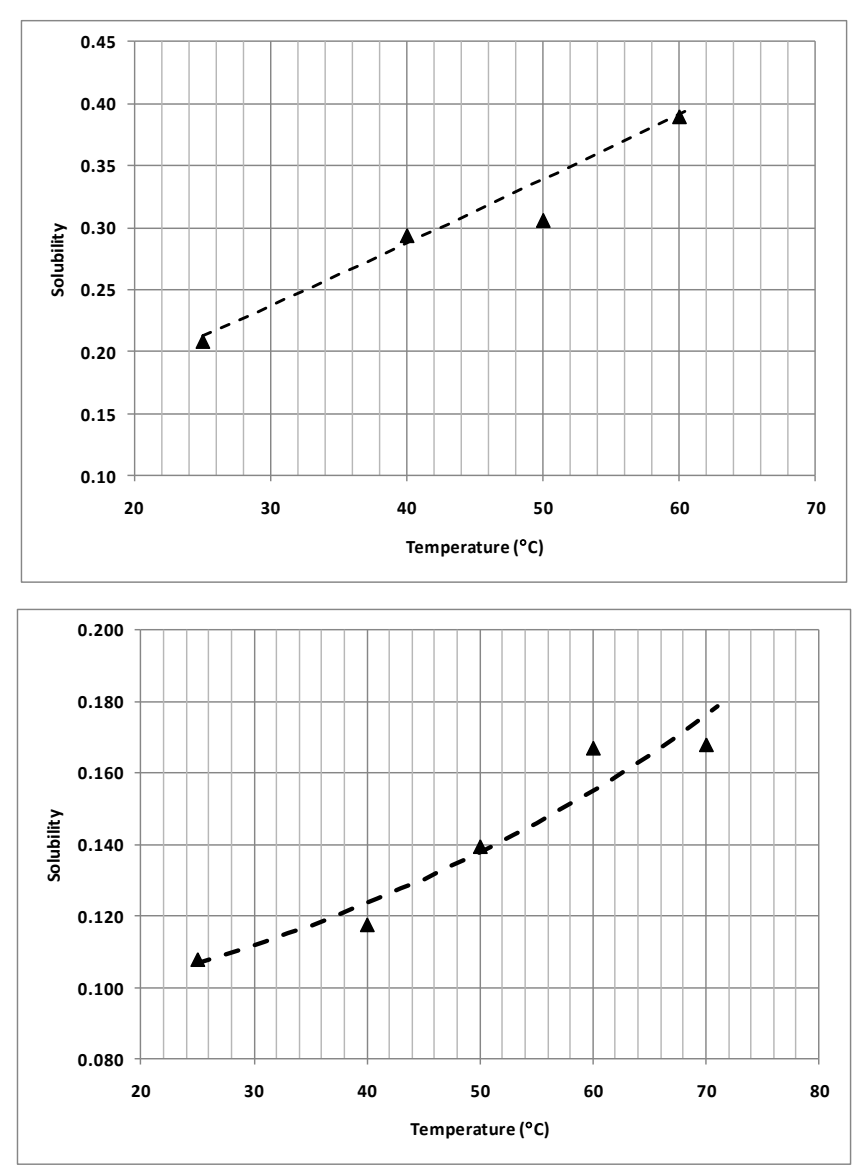

Fig. 2. NRTL model $v s$ exp. solubilities of glucose in IL2 (in the top) and IL4.

\section{CONCLUSION}

It was found that the solubility of glucose and fructose in ILs depends on the type of the anion, cation, and substituents on the cation. However, the anion plays the most important 
role. Depending on the type of the anion, some ILs dissolve more fructose than glucose while others dissolve more glucose than fructose. A process for separation of mixed dried glucose and fructose into glucose and fructose solid precipitate and/or enriched solutions of either of glucose or fructose was proposed. By the virtue of said difference in solubility, the proposed process is capable of separation of glucose and fructose feed into $99 \%$ yield of respective pure component. This separation process has very important applications in industry. Glucose solubility variation with temperature for two ionic liquids has been modeled using the NRTL activity coefficient model. Comparison with experimental data is quite satisfactory.

\section{REFERENCES}

[1] R. Rinaldi and F. Schüth, "Design of solid catalysts for the conversion of biomass," Energy Environ. Sci., vol. 2, pp. 610-626, 2009.

[2] D. Langev, M. Metayer, M. Labbe, and C. Chappey, "Electromembrane process for sugar separation: a preliminary study," Russ. J. Electrochem, vol. 32, pp. 241-247, 1996.

[3] C. C. Akoh and B. G. Swanson, Carbohydrate polyesters as fat substitutes, Dekker Publisher, New York, 1994.

[4] M. Heper, L. Turker, and N. S. Kincal, "Sodium, ammonium, calcium, and magnesium forms of zeolite $\mathrm{Y}$ for the adsorption of glucose and fructose from aqueous solutions," J. of Colloid and Interface Science, vol. 306, pp. 11-15, 2007.

[5] S. Kishihara, S. Fuji, S. Tamaki, K. B. Kim, T. Wakiuchi, and T. Yamamoto, "Continuous separation of sucrose, glucose and fructose using simulated moving bed adsorber," Int. Sugar J., vol. 94, pp. 305-308, 1992.

[6] K. N. Lee and W. K. Lee, "Model for the separation of glucose and fructose at high concentration using a semi-continuous chromatographic refiner," J. Chem Eng. Jpn., vol. 25, pp. 533-538, 1992.

[7] Lj. Matijaševi'c and D. Vasi'c-Ra cki, "Separation of glucose/fructose mixtures: counter-current adsorption system," Biochemical Engineering Journal, vol. 4, no. 2, pp. 101-106, 2000.

[8] R. M. Lau, F. van Rantwijk, K. R. Seddon, and R. Sheldon, "Lipase-catalyzed reactions in ionic liquids," Org. Lett., vol. 2, pp. 4189-4191, 2000.

[9] S. Park and R. J. Kazlauskas, "Improved preparation and use of room-temperature ionic liquids in lipase-catalyzed enantio- and region-selective acylations," J. Org. Chem., vol. 66, pp. 8395-8401, 2001.

[10] N. Kimizuka and T. Nakashima, "Spontaneous self-assembly of glycolipid bilayer membranes in sugar-philic ionic liquids and formation of ionogels," Langmuir, vol. 17, pp. 6759-6761, 2001.

[11] D. R. MacFarlane, J. Golding, S. Forsyth, M. Forsyth, and G. B. Deacon, "Low viscosity ionic liquids based on organic salts of the dicyanamide anion," Chem. Commun, vol. 16, pp. 1430-1431, 2001.

[12] S. A. Forsyth, D. R. MacFarlane, R. J. Thomson, and M. von Itzstein, "Rapid, clean, and mild O-acetylation of alcohols and carbohydrates in an ionic liquid," Chem. Commun, vol. 7, pp. 714-715, 2002.

[13] J. Pernak, A. Olszowka, and R. Olszewski, "New Ionic Liquids with Alkoxymethyl Hydrophobic Groups," Pol. J. Chem., vol. 77, pp. 179-187, 2003.

[14] Q. B. Liu, M. H. A. Janssen, F. van Rantwijk, and R. A. Sheldon, "Room-temperature ionic liquids that dissolve carbohydrates in high concentrations," Green Chem., vol. 7, pp. 39-42, 2005.
[15] A. A. Rosatella, L. C. Branco, and C. A. M. Afonso, "Studies on dissolution of carbohydrates in ionic liquids and extraction from aqueous phase," Green Chem., vol. 11, pp. 1406-1413, 2009.

[16] H. Zhao, C. L. Jones, and J. V. Cowins, "Lipase dissolution and stabilization in ether-functionalized ionic liquids," Green Chem., vol 11, no. 8, pp. 1128-1129, 2009

[17] H. Zhao, G. A. Baker, Z. Song, O. Olubajo, T. Crittle, and D. Peters, "Designing enzyme-compatible ionic liquids that can dissolve carbohydrates," Green Chem., vol. 10, pp. 696-705, 2008.

[18] S. H. Lee, D. T. Dang, S. H. Ha, W. J. Chang, and Y. M. Koo, "Lipase-catalyzed synthesis of fatty acid sugar ester using extremely supersaturated sugar solution in ionic liquids," Biotechnol. Bio Eng., vol. 99, pp. 1-8, 2008

[19] J. M. Prausnitz, R. N. Lichtenthaler, and E. G. de Azevedo, Molecular Thermodynamics of Fluid-Phase Equilibria, $3^{\text {rd }}$ ed., Prentice Hall International, 1999.

[20] S. L. Sandler, Chemical and Engineering Thermodynamics, $3^{\text {rd }}$ ed., John Wiley \& sons, 1999.

[21] O. Ferreira, E. A. Brignole, and E. A. Macedo, "Phase equilibria in sugar solutions using the A-UNIFAC model," Ind. Eng. Chem. Res. vol. 42, pp. 6212-6222, 2003.

[22] H. Renon and J. M. Prausnitz, "Local compositions in thermodynamic excess functions for liquid mixtures," AIChE Journal, vol. 14, no. 3, pp. 135-144, 1968.

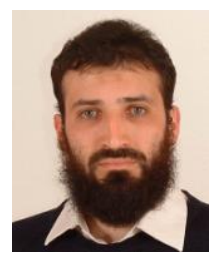

M. K. Hadj-Kali was born in Algeria in 1977. He received his BS in chemical engineering, with first class honors, from École Nationale Polytechnique (ENP), Algiers, in 1999. In 2004, He got his PhD in chemical engineering from Institut National Polytechnique de Toulouse (INPT), France.

He worked at École Nationale Superieure des Arts Chimiques Et Technologiques (ENSIACET), Toulouse, from 2004 to 2006 and then he occupied a post-doctoral position from 2006 to 2008 working on the phase equilibria related to the iodine-sulfure thermochemical cycle for hydrogen production.

Dr. Hadj-Kali is currently an assistant professor at King Saud University since 2009. Dr. Hadj-Kali has co-authored more than 20 peer-reviewed journal publications and his research activities are focused on green solvents applications and fluid phase equilibria modeling using methods based on both statistical and classical thermodynamics.

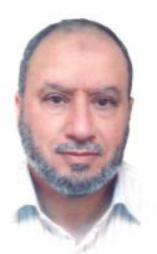

I. M. AlNashef obtained his Ph. D. in 2004 from the University of South Carolina. Then, he joined King Saud University (Riyadh, Saudi Arabia) as an assistant professor. In 2011, Dr. AlNashef was promoted to associate professor.

$\mathrm{He}$ is very active in research related to green engineering and sustainability. He established collaboration with University of Malaya, KL, Malaysia, where he was a co-advisor for seven $\mathrm{Ph}$. D. students. He moved to Abu Dhabi (UAE) where he is now employed as an associate professor in the Department of Chemical and Environmental Engineering at Masdar Institute of Science and Technology (MIST).

Dr. AlNashef co-authored more than 60 peer-reviewed journal publications. In addition, he received 7 patents from US and EU Patent Offices. He is also a recipient of several prestigious awards including King Abdullah award for best invention in 2013. He supervised 9 graduate students. 